\title{
Very short-term conceptual memory
}

\author{
MARY C. POTTER \\ Massachusetts Institute of Technology, Cambridge, Massachusetts
}

\begin{abstract}
Short-term memory for conceptual information is largely missing from current models of shortterm memory. Several phenomena are discussed that give evidence for very brief conceptual representations of stimuli. Although these fleeting representations do not surface readily with many of the standard methods for studying and testing short-term memory, I argue that they are fundamental to cognitive processing and to the form that long-term memory takes.
\end{abstract}

The thesis of this paper is that fleeting conceptual representations arise early in perceptual processing and are ubiquitous in perception, memory retrieval, and thought. They constitute a form of very short-term memory (CSTM) that is conceptually structured and tightly linked to longterm memory (LTM), rather than being simply iconic or echoic. Conceptual STM, called CSTM hereafter, is distinct from short-term memory (STM) as measured by the memory span (e.g., Miller, 1956; see Schweickert, 1993).

Unlike STM, CSTM is central to cognitive processing. Recognition of meaningful stimuli such as words or objects rapidly activates conceptual information and leads to the retrieval of additional relevant information from LTM. New links among concurrently active concepts are formed, guided by parsing mechanisms of language or scene perception and by higher level knowledge. When these new links result in well-connected structures, the structures are likely to be consolidated into LTM. Information that is not incorporated into such structures is rapidly forgotten. This whole cycle-identification of stimuli, memory recruitment, structuring, consolidation, and forgetting of nonstructured material-may occur in less than $1 \mathrm{sec}$ when viewing a pictured scene or reading a sentence.

Figure 1 shows a rough diagram of the architecture I am proposing for reading and other visual activities; aural understanding would be expected to be similar in most respects. The boxes in Figure 1 may or may not correspond to distinct processing modules, as we shall see. A visual stimulus, which in Figure 1 is a short sentence, is identified by steps not detailed in the figure, with conceptual output to CSTM and phonological output to conventional STM. STM consists of the phonological representation, coupled with an articulatory loop that permits

\footnotetext{
A version of this paper was presented as part of the symposium, "Short-term memory: Where do we stand?," at the 32nd Annual Meeting of the Psychonomic Society, San Francisco, November 1991. This research was supported by National Science Foundation Grants BNS8318156, BNS86-19053, and BNS90-13026 to M.C.P. I thank my many collaborators and research assistants who participated in the research described. I also thank Alan Baddeley, Robert Crowder, Judith Kroll, and Virginia Valian for their helpful comments. Requests for reprints should be sent to M.C.P., Department of Brain and Cognitive Sciences, E10-039, Massachusetts Institute of Technology, Cambridge, MA 02139.
}

rehearsal (see Baddeley, 1986, 1992). Immediate, overt recall may be based on the articulatory-loop system. Without continued rehearsal, the contents of STM either decay or are lost through interference over a period of several seconds (see Schweickert, 1993, for a model). Rehearsal in STM plays little or no direct role in establishing a long-term representation, other than a phonological or articulatory one, except insofar as the material is reheard by the internal "ear," recognized again, and reentered into CSTM for "deeper" processing (Craik \& Lockhart, 1972; cf. Shiffrin, 1993). With some important exceptions, among them the learning of spoken language, neither the phonological buffer nor rehearsal plays a significant role in most processing outside the laboratory (Baddeley, 1986; Martin, 1993). ${ }^{1}$

CSTM, in contrast, is a major component of the higher level processing important for retention in LTM. As conceived here, the contents of this memory include not only elements that are activated and copied from LTM during stimulus identification, but also new connections among some of these elements that are made on the fly. Much more information is present in CSTM than will be retained; in general, only the part of the information that can be organized immediately into a meaningful structure will persist more than momentarily. That is, the same principles govern persistence in LTM and in CSTM. Figure 1 is deliberately ambiguous about whether CSTM is an activated part of LTM that includes new but sometimes ephemeral connections or a separate episodic representation that passes information to and from LTM (this issue is discussed below). In either case, CSTM is part of a dynamic, structure-building process rather than a passive store.

Finally, Figure 1 indicates that short-term recall may be based not only on STM but also on new LTM representations. In the latter case, recall will involve regeneration from an abstracted conceptual representation rather than readout from the phonological representation of STM. I will have more to say about regeneration in recall shortly.

Two decades ago, it was still a matter of debate whether semantic coding occurred in short-term memory or only subsequent to short-term memory. For example, Shulman (1971) concluded in a review that phonemic encoding is 


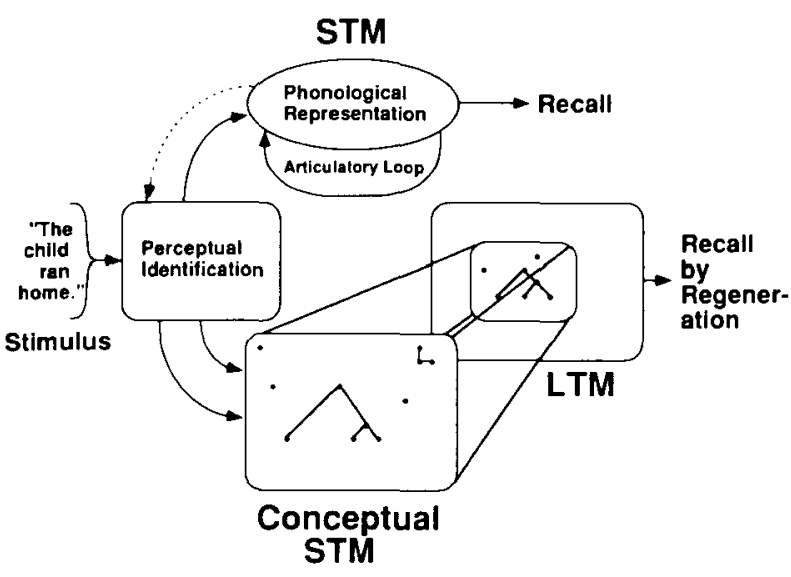

Figure 1. Architecture of memory.

faster than semantic encoding and thus "encoding will be primarily phonemic in short-term memory unless task demands require semantic encoding"' (Abstract, p. 399). It is now widely accepted, however, that conceptual (semantic) information about a stimulus such as a word or a picture is available rapidly. Evidence has come from many sources, among them semantic priming (e.g., Hines, Czerwinski, Sawyer, \& Dwyer, 1986; Marcel, 1983; Meyer \& Schvaneveldt, 1971; see Neely, 1991), speech shadowing (e.g., Marslen-Wilson, 1975), eye fixation studies (e.g., Ehrlich \& Rayner, 1981; see also Potter, 1983 , and other chapters in Rayner, 1983), target search (e.g., Biederman, 1972), and studies measuring eventrelated potentials (ERP; e.g., Kutas \& Hillyard, 1980). Furthermore, not only is conceptual information activated rapidly, but it may be forgotten equally rapidly. The studies that I will focus on, therefore, are ones in which a viewer is momentarily aware of a stimulus but may forget it almost immediately when additional stimuli are processed. This situation mimics normal steady-state processing, in which the flow of stimuli or of thoughts is apt to be continuous, and may be contrasted with the study of single stimuli presented on separate trials.

In these studies, a rapid sequence of items is presented, such as a sequence of unrelated pictures. The logic requires one to show that the pictures are momentarily understood but then are likely to be quickly forgotten-much more quickly than they are forgotten if they are presented at rates similar to those used in conventional short-term memory studies. In a series of experiments with such sequences of pictures (Potter, 1975, 1976; Potter \& Levy, 1969), we contrasted two tasks. One required immediate semantic detection; for example, subjects had to respond when they saw a picture of "a picnic" (which they had never seen before), presented in a sequence of 16 pictures. Most targets were successfully detected, even at a rate of 8 pictures/sec. In the other task, a different group of subjects was given a recognition test after each sequence. Here, although recognition memory was excellent when the pictures were presented for 1 or 2 sec each (cf. Standing, 1973), at higher rates there was a rapid drop-off in recognition memory; performance was not much above chance at 8 pictures/sec. Thus, there was a marked difference in time course between initial picture identification (required for detection of the conceptually specified target) and memory storage. We concluded that retention for even a relatively short time requires additional processing - consolidation-beyond that required for initial understanding. These results and others (e.g., Intraub, 1981, 1984) strongly suggest that pictured scenes are understood within about $100 \mathrm{msec}$, but (when presentation is continuous) many are then forgotten within a few hundred milliseconds, which supports the central claim of the present model.

A similar method for presenting sequences of words was dubbed rapid serial visual presentation (RSVP) by Forster (1970). Unlike pictures, unrelated words are difficult to remember if they exceed the memory span, even when each is shown for 1 or $2 \mathrm{sec}$. We therefore looked at the effect of rate of presentation on short lists. Lists of 2 to 6 unrelated nouns (followed by a mask) were presented at rates between 1 and 12 words/sec for immediate recall (Potter, 1982). As Figure 2 shows, the 2-word list was near the ceiling at all rates, indicating that the words could be identified even at the highest rate. But a mean of only 2.6 words, well under the standard memory span, was the most that could be recalled for any list length at the highest rate. Only when the rate fell to about 3 words/sec did performance reach that of the standard memory span. (Words are presented at 1 per second in a standard span test.)

This result indicates that at high rates, words may be perceived and then quickly forgotten without entering conventional STM. It does not prove, however, that the words were understood briefly; possibly an iconic or orthographic representation was sufficient to support recall of the 2 or 3 words that could be reported. Experiments with RSVP sentences show, however, that words can be readily understood at such rates. In marked contrast to the experiment with lists of unrelated words, an RSVP sentence of 14 words can be parsed, understood, and immediately recalled with near-perfect accuracy when the rate of presentation is as high as 10 or 12 words/sec (Potter, Kroll, Yachzel, Carpenter, \& Sherman, 1986; for a review of RSVP research, see Potter, 1984). The contrast between sentences and lists in our experiments implies that sentence structure and meaning are recovered as the words are viewed; conventional STM would not be able to register and retain the words so that the sentence could be processed later.

Returning to the model in Figure 1, notice again that there are two modes of immediate recall, one from the phonological store (conventional STM) and one from the recently activated part of LTM, where the representation is of meanings abstracted from the surface stimulus. Lombardi and I (Lombardi \& Potter, 1992; Potter \& Lombardi, 1990) proposed that immediate recall of sentences that are longer than the single-word memory span involves 


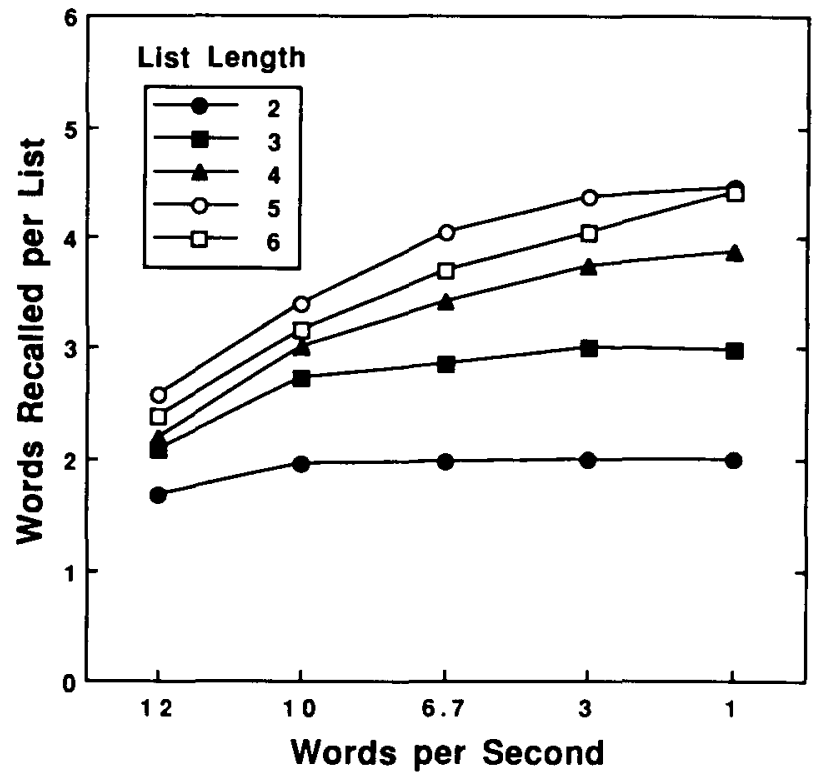

Figure 2. Immediate recall of RSVP (rapid serial visual presentation) lists of $2,3,4,5$, or 6 nouns presented at rates between 1 and 12 words/sec (Potter, 1982). A mask followed each list.

regeneration from such a conceptual representation. In expressing the propositional structure of the sentence, the recaller preferentially selects lexical items that have been recently activated, and the result is that recall is often verbatim even though there is no verbatim memory trace. Consistent with this hypothesis, subjects made many in trusion errors in immediate recall of sentences when a synonymous lure word was presented in a secondary task. ${ }^{2}$ Performance was similar whether the to-be-recalled sentence was presented at 10 words/sec or at the more normal reading speed of 5 words $/ \mathrm{sec}$, again supporting the claim that sentences can be understood and their meanings retained at least briefly at rates of presentation never contemplated in earlier theories of STM.

Two other phenomena from our laboratory also provide evidence for rapid comprehension: nonword conversion and double-word selection. In both sets of experiments, subjects are asked to read and immediately recall sentences presented serially at 7 to 10 words $/ \mathrm{sec}$. In the nonword procedure, a nonword that is one letter away from each of 2 words is substituted for one of those words in the sentence, as illustrated in Table 1. When reading at 10 words/sec, the subjects fairly frequently misread the nonword as a word (Potter, Moryadas, Abrams, \& Noel, 1993). Furthermore, sentence context can exert a strong influence on which word the nonword converts to $\mathrm{Ta}$ ble 2), even though that word is not highly predictable from the context alone. Clearly, the meaning of the sentence context can be processed rapidly enough to interact with (mis)identification of the nonword.

In another condition in this experiment, a wrong word (rather than the nonword) was substituted (e.g., duck instead of deck, or vice versa). Again, this word was often misread, as Table 2 shows. The subjects were warned to expect some nonwords and nonsensical sentences and to report them as seen, so their misreadings were probably misperceptions rather than intentional corrections. The rapid and unconscious convergence on a context-relevant word that is orthographically similar to the stimulus suggests that sets of similar words (and their meanings) are activated briefly in the process of word identification, as McClelland and Rumelhart (1981) and others have proposed.

The second phenomenon of this type (see Potter, Moryadas, \& Stiefbold, 1992) required subjects to make a rapid selection from two actual words presented simultaneously at one point in an RSVP sentence, as illustrated in Table 3. In this experiment, the subjects read at 7.5 words $/ \mathrm{sec}$ (133 msec/word), but the critical word pair appeared for only $83 \mathrm{msec}$. The subjects were surprisingly successful in picking the correct word to include in their recall of the sentence, as Table 4 shows. More striking, they typically forgot (or never became conscious of) the word that had not been selected, even though we asked them to report it after recalling the sentence. (Both words were seen and retained long enough to allow context, which was provided only after the critical pair, to have some influence on selection, however.) This result provides direct evidence for the rapid availability of the conceptual representation of a word, followed by rapid forgetting when that word is irrelevant to the higher level structure.

\section{Relation of Conceptual STM \\ to Existing Theories of Memory}

Current accounts of short-term memory acknowledge shortcomings of the modal memory model (Baddeley, 1986; cf. Shiffrin, 1993) and replace it with an eclectic

Table 1

Example of a Word-Sentence Set for the Critical Words duck and deck and Nonword dack

\begin{tabular}{cl}
\hline Sentence Bias & \multicolumn{1}{c}{ Sentence and Nonword } \\
\hline Duck & The child fed the dack at the pond. \\
Deck & The sailor washed the dack of that vessel. \\
Neutral & The visitors noticed the dack by the house. \\
\hline
\end{tabular}

Note-Adapted from Potter et al. (1993).

Table 2

Percentage of Responses

\begin{tabular}{lcc}
\hline & \multicolumn{2}{c}{ Response } \\
\cline { 2 - 3 } Sentence & Word A & Word B \\
\hline & Nonword Presented & \\
Biased $\rightarrow$ B & 3 & 40 \\
Neutral & $12^{*}$ & 12 \\
& Word A Presented & \\
Biased $\rightarrow$ B & $46 \dagger$ & 26 \\
Neutral & $68 \dagger$ & 3 \\
\hline
\end{tabular}

Note-Adapted from Potter et al., 1993. The percentages in each row are based on 512 trials. "Word A" is defined as the word presented on a given trial, "Word B"' as the word biased toward, on biased trials. *Nonword conversions to words $A$ and $B$ were averaged, since there was no principled difference between them in the neutral condition. tCorrect response. 
Table 3

Double-Word Sentences With Bias Before or After the Double-Word Array*

Before

Maggie wrote the letter with $a_{* * * * *}^{\text {basket }}$ she had with her. pencil

Maggie carried the kitten in a basket/pencil to her house.

After

Maggie used a basket/pencil to write the letter.

Maggie used a basket/pencil to carry the kitten.

*The first sentence indicates the form of the double-word array. The sentence was shown using RSVP; subjects were instructed to recall the sentence with the appropriate word from the double-word array, reporting the other word after the sentence

Table 4

Percent Recall of the Matching and Mismatching Double-Word in Each Condition

\begin{tabular}{lccccc}
\hline & \multicolumn{2}{c}{ Recalled in Sentence } & & \multicolumn{2}{c}{ All Recalls* } \\
\cline { 2 - 3 } \cline { 5 - 6 } Context & Match & Mismatch & & Match & Mismatch \\
\hline Before & 75 & 11 & & 75 & 16 \\
After & 65 & 15 & & 66 & 22 \\
Both & 70 & 13 & & 70 & 19
\end{tabular}

*Including recall after the sentence.

framework termed "working memory." This framework allows for a variety of components, including the articulatory loop system, which I refer to as STM, and a visuospatial sketchpad (Baddeley, 1986), which I have not discussed here. In most current models, the memory associated with rapid conceptual processing (if such a memory is even considered) is subsumed under semantic priming or persistence of activation (see Cowan, 1993). Short-term memory for conceptual information cannot simply be the activation of concepts and connections already in long-term memory, however, because such reactivation does not allow for the new configurations and interpretations necessary to encode almost any experience. Moreover, if the new event is to be remembered, it must be marked as a distinctive episode.

An obvious case is the representation of the meaning of a new sentence (and most sentences we encounter are new). It is not enough just to activate the type representation of each word in LTM; one must set up an episodic representation that includes a token of each word type, tokens that are pointers to or copies of the long-term representation of the words and their meanings (Kahneman \& Treisman, 1984, proposed a similar idea for the perception of an object, the opening of a temporary object file). It is over this new representation that parsing and message-level processing takes place, resulting in the formation of additional, episode-specific links among the original LTM concepts. As this new episodic representation is formed, it is registered in LTM, where it may be lost rapidly or slowly, or may last a lifetime. 3
Thus, returning to Figure 1, one sees that the conceptual STM box represents more than a close-up of an active part of preexisting LTM. The new and old connections that are so rapidly set up as one reads a sentence at 10 words/sec constitute a functionally distinct pattern that has ties to the time, place, and setting. In this view, CSTM consists of tokens or copies of LTM types and their associations, together with new associations among these tokens and the episodic context. Interestingly, creating a second token of a given type is a rate-limited process, so that the second occurrence of a word may fail to be assigned a distinct token at high rates of presentation and will simply disappear. This phenomenon, called repetition blindness, was first studied by Kanwisher $(1987,1991$; Kanwisher \& Potter, 1989, 1990). The fact that visual, orthographic, or phonological similarity (Bavelier \& Potter, 1992) can each produce repetition blindness, but conceptual similarity apparently cannot (Kanwisher \& Potter, 1990), suggests that nonconceptual properties of stimuli play a special role in establishing tokens in CSTM.

\section{Other Experimental Work Relevant to CSTM}

Work in a number of domains that have been considered separate from each other are relevant to CSTM. I have already noted that semantic priming experiments, eye movement research, and many other studies give evidence for rapid access to stimulus meaning. Two other areas-research on iconic memory and studies of ambiguity resolution in language processing-also deserve mention. Iconic memory, as measured by partial report superiority in Sperling's (1960) study and subsequent work, was taken initially to reflect a sensory, precategorical form of very short-term memory. More recent work (reviewed by Coltheart, 1983) has suggested that partial report superiority does not depend on the icon, but on a subsequent representation that is postcategorical but shortlasting. I speculate that these representations are part of CSTM, even though the random letters and digits used in such studies are conceptually impoverished.

Theories of ambiguity resolution in language typically assume the existence of nonconscious (or only briefly conscious) conceptual representations. For example, Swinney (1979) proposed that the resolution of lexical ambiguity depends on the unconscious activation of all the meanings of the ambiguous word, followed within a few hundred milliseconds by the correct (context-relevant) choice of meaning. MacDonald, Just, and Carpenter (1992) and others have provided evidence that there is parallel computation of alternative structures for locally syntactically ambiguous sentences. Whether these multiple alternatives are represented momentarily in CSTM, or whether they are represented in an earlier, nonconscious part of processing, is an open question.

\section{Conclusion}

I have argued that there is a cognitively important stage of processing that occurs well before STM and that has associated with it a fleeting form of memory: conceptual 
very short-term memory, or CSTM. CSTM is the basis for LTM; material that becomes conceptually wellstructured as it is processed in CSTM is likely to persist at least briefly in LTM, permitting (for example) regeneration of an RSVP sentence in immediate recall. Conventional STM, in contrast, is a largely separate system, a buffer that can maintain phonological representations (see Baddeley, 1986). In what ways CSTM and STM representations interact during processing and in immediate memory tasks remains to be elucidated. Although I suggest that CSTM is a neglected stage of memory, in fact many investigators are already studying it, whatever they may call it. A much clearer understanding of early processing is likely to evolve in the next decade as the nature of short-term memory is redefined.

\section{REFERENCES}

Baddeley, A. (1986). Working memory. Oxford: Clarendon. BADDELEY, A. (1992). Working memory. Science, 255, 556-559.

BAvelier, D., PotTER, M. C. (1992). Visual and phonological codes in repetition blindness. Journal of Experimental Psychology: Human Perception \& Performance, 18, 134-147.

Biederman, I. (1972). Perceiving real-world scenes. Science, 177 77-80

Besner, D., \& DavelaAr, E. (1982). Basic processing in reading: Two phonological codes. Canadian Journal of Psychology, 36, 701-711.

Coltheart, M. (1983). Iconic memory. Philosophical Transactions of the Royal Society of London, B302, 283-294.

Cowan, N. (1993). Activation, attention, and short-term memory. Memory \& Cognition, 21, 162-167.

Craik, E. I. M., \& LockHART, R. S. (1972). Levels of processing: A framework for memory research. Journal of Verbal Learning \& Verbal Behavior, 11, 671-684.

EhrLICH, S. F., \& RAYNER, K. (1981). Context effects on word perception and eye movements during reading. Journal of Verbal Leaming \& Verbal Behavior, 20, 641-655.

FORSTER, K. I. (1970). Visual perception of rapidly presented word se quences of varying complexity. Perception \& Psychophysics, 8 , 215-221.

Hines, D., Czerwinski, M., SAWyer, P. K., DWyer, M. (1986) Automatic semantic priming: Effect of category exemplar level and word association level. Journal of Experimental Psychology: Human Perception \& Performance, 12, 370-379.

INTRAUB, H. (1981). Rapid conceptual identification of sequentially presented pictures. Journal of Experimental Psychology: Human Percep tion \& Performance, 7, 604-610.

INTRAUB, H. (1984). Conceptual masking: The effects of subsequent visual events on memory for pictures. Joumal of Experimental Psychology: Learning, Memory, \& Cognition, 10, 115-125.

Kahneman, D., \& Treisman, A. M. (1984). Changing views of attention and automaticity. In R. Parasuraman \& D. R. Davies (Eds.), Varieties of attention (pp. 29-61). New York: Academic Press.

KANWIShER, N. G. (1987). Repetition blindness: Type recognition without token individuation. Cognition, 27, 117-143.

KANWISHER, N. G. (1991). Repetition blindness and illusory conjunctions: Errors in binding visual types with visual tokens. Journal of Experimental Psychology: Human Perception \& Performance, 17, 404-421.

Kanwisher, N., \& Potter, M. C. (1989). Repetition blindness: The effects of stimulus modality and spatial displacement. Memory \& $\mathrm{Cog}$ nition, 17, 117-124.

Kanwisher, N. G., \& Potter, M. C. (1990). Repetition blindness: Levels of processing. Journal of Experimental Psychology: Human Perception \& Performance, 16, 30-47.
Kutas, M., \& Hillyard, S. A. (1980). Reading senseless sentences: Brain potentials reflect semantic incongruity. Science, 207, 203-205.

Lombard, L., \& Potter, M. C. (1992). The regeneration of syntax in short term memory. Journal of Memory \& Language, 31, 713-733.

MacDonald, M. C., Just, M. A., \& Carpenter, P. A. (1992). Working memory constraints on the processing of syntactic ambiguity. $C o g$ nitive Psychology, 24, 56-98.

MarCEL, A. J. (1983). Conscious and unconscious perception: Experiments on visual masking and word recognition. Cognitive Psychology, 15, 197-237.

MARSLEN-WILSON, W. D. (1975). Sentence perception as an interactive parallel process. Science, 189, 226-228.

Martin, R. C. (1993). Short-term memory and sentence processing: Evidence from neuropsychology. Memory \& Cognition, 21, 176-183.

MCClelland, J. L., \& Rumelhart, D. E. (1981). An interactive model of context effects in letter perception: Part 1. An account of basic findings. Psychological Review, 88, 375-407.

Meyer, D. E., \& SChVANeveldt, R. W. (1971). Facilitation in recognizing pairs of words: Evidence of a dependence between retrieval operations. Journal of Experimental Psychology, 90, 227-234.

Miller, G. A. (1956). The magical number seven, plus or minus two: Some limits on our capacity for processing information. Psychological Review, 63, 81-97.

NeELY, J. H. (1991). Semantic priming effects in visual word recognition: A selective review of current findings and theories. In D. Besner \& G. W. Humphreys (Eds.), Basic processes in reading: Visual word recognition (pp. 264-336). Hillsdale, NJ: Erlbaum.

Perfetri, C. A., \& MCCutchen, D. (1982). Speech processing in reading. Speech \& Language: Advances in Basic Research \& Practice, 7, 237-269.

PotTeR, M. C. (1975). Meaning in visual search. Science, 187, 965-966.

PotTER, M. C. (1976). Short-term conceptual memory for pictures. Journal of Experimental Psychology: Human Learming \& Memory, 2, 509-522.

Potter, M. C. (1982, November). Very short-term memory: In one eye and out the other. Paper presented at the 23rd Annual Meeting of the Psychonomic Society, Minneapolis.

PotTer, M. C. (1983). Representational buffers: The eye-mind hypothesis in picture perception, reading, and visual search. In K. Rayner (Ed.), Eye movements in reading: Perceptual and language processes (pp. 413-437). New York: Academic Press.

PotTER, M. C. (1984). Rapid serial visual presentation (RSVP): A method for studying language processing. In D. Kieras \& $M$. Just (Eds.), New methods in reading comprehension research, (pp. 91118). Hillsdale, NJ: Erlbaum.

Potter, M. C., Kroll, J. F., \& Harris, C. (1980). Comprehension and memory in rapid sequential reading. In R. Nickerson (Ed.), Attention and Performance VIII (pp. 395-418). Hillsdale, NJ: Erlbaum.

Potter, M. C., Kroll, J. F., Yachzel, B., Carpenter, E., SherMAN, J. (1986). Pictures in sentences: Understanding without words. Journal of Experimental Psychology: General, 115, 281-294.

Potrer, M. C., \& LEVY, E. I. (1969). Recognition memory for a rapid sequence of pictures. Journal of Experimental Psychology, 81, 10-15.

PotTer, M. C., \& LombARDI, L. (1990). Regeneration in the shortterm recall of sentences. Joumal of Memory \& Language, 29, 633-654.

Potter, M. C., Moryadas, A., Abrams, I., \& Noel, A. (1993). Word perception and misperception in context. Journal of Experimental Psychology: Learning, Memory, \& Cognition, 19, 3-22.

Potter, M. C., Moryadas, A., \& Stiefbold, D. R. (1992). Word selection in reading sentences: Preceding versus following contexts. Unpublished manuscript, MIT, Cambridge, MA.

RAYNER, K. (ED.) (1983). Eye movements in reading: Perceptual and language processes. New York: Academic Press.

SCHWEICKERT, R. (1993). A multinomial processing tree model for degradation and redintegration in immediate recall. Memory \& Cognition, 21, 168-175.

Shiffrin, R. M. (1993). Short-term memory: A brief commentary. Memory \& Cognition, 21, 193-197.

Shulman, H. G. (1971). Similarity effects in short-term memory. Psychological Bulletin, 75, 399-415. 
SPERLING, G. (1960). The information available in brief visual presentations. Psychological Monographs, 74(11. Whole No. 498).

StandiNG, L. (1973). Learning 10,000 pictures. Quarterly Journal of Experimental Psychology, 25, 207-222.

SwINNEY, D. A. (1979). Lexical access during sentence comprehension: (Re)consideration of context effects. Journal of Verbal Learning \& Verbal Behavior, 18, 645-659.

\section{NOTES}

1. Although retrieval of the phonological representation of a written word does play an important role in fixing its identity or reference (Perfetti \& McCutchen, 1982), this "early" phonological representation is not the one implicated in the conventional short-term span (see Bavelier \& Potter, 1992; Besner \& Davelaar, 1982).

2. Martin (1993) describes a patient, E.A., who has a severely impaired phonological short-term memory and makes numerous paraphrase errors in recalling sentences. Martin concludes that this finding shows that STM plays a role in normal verbatim sentence recall, contrary to
Potter \& Lombardi's ( 1990 ) claim. Another possibility, however, is that lexical activation is impaired in $\mathbf{E}$. A. so that she is unable to select only recently encountered words when regenerating a sentence. In any case. E. A. 's ability to understand sentences and to base her immediate recall on meaning, without the use of STM. suppors our basic claim that sentence meaning is computed directly and does not depend on STM.

3. Consolidation in LTM normally requires that attentional processing continue for several hundred milliseconds after initial identification and structuring (Potter, 1975. 1976: Potter, Kroll, \& Harris, 1980). Such added processing occurs automatically if (1) events or stimuli to which the viewer is attending change at a slow rate. (2) stimuli change rapidly but can be incorporated immediately into a more slowly chang. ing higher level representation, or (3) a given item is selected for additional processing (at the expense of other stimuli). Otherwise, the contents of CSTM quickly decay or are overwritten.

(Manuscript received April 2, 1992:

revision accepted for publication September 2, 1992.) 